Original

\title{
Verification of osteoblast differentiation on airborne-particle abrasion, large-grit, acid-etched surface of titanium implants regulated by yes-associated protein and transcriptional coactivator with PDZ-binding motif
}

\author{
Ting Wei1,2), Jiayi $\mathrm{Li}^{3)}$, Huiqiang Sun ${ }^{1,2)}$, Mengyang Jiang1), Yun Yang1), \\ Xiayan Luo ${ }^{1)}$, and Tingsong Liu ${ }^{1)}$ \\ 1)Department of Prosthodontics, School of Stomatology, Shandong University, Jinan, P. R. China \\ 2)Shandong Provincial Key Laboratory of Oral Tissue Regeneration, School of Stomatology, \\ Shandong University, Jinan, P. R. China \\ 3)Department of Oral and Maxillofacial-Head Neck Oncology, Ninth People's Hospital, \\ Shanghai Jiao Tong University School of Medicine, Shanghai, P. R. China
}

(Received April 1, 2018; Accepted November 22, 2018)

\begin{abstract}
Although airborne-particle abrasion, large-grit, acid-etched (SLA) surface technology can promote implant osseointegration; its mechanism remains unclear. By preparing the SLA titanium (Ti) plate (SLA Ti) and Polished Ti plate (Polished Ti), this experiment investigates the expression and distribution of the Yes-associated protein (YAP) and transcriptional coactivator with the PDZ-binding motif (TAZ) in MC3T3-E1 cells. In addition, gene YAP and TAZ silencing on the SLA Ti was conducted to observe changes in the osteoblast differentiation markers, runt-related transcription factor-2 (Runx2) and bone sialoprotein (BSP). The results demonstrated that SLA Ti surface microtopography could induce YAP/TAZ's transfer from the cytoplasm to the nuclei of MC3T3-E1 cells. The expression of YAP/TAZ increased in terms of mRNA and protein. After silencing the YAP/TAZ genes, Runx2 and
\end{abstract}

Correspondence to Dr. Huiqiang Sun, Department of Prosthodontics, School of Stomatology, Shandong University, Culture West Road 44-1, Jinan, Shandong 250012, P. R. China Fax:+86-531-88382058 E-mail: whitedove69@163.com

J-STAGE Advance Publication: July 20, 2019

Color figures can be viewed in the online issue at J-STAGE.

doi.org/10.2334/josnusd.18-0112

DN/JST.JSTAGE/josnusd/18-0112
BSP decreased, suggesting that YAP/TAZ plays an important regulatory role in this process. Meanwhile, the results also showed that SLA microtopography enhanced the expression of integrins $\alpha 1, \alpha 2$, and $\beta 1$. After silencing the integrin $\alpha 1, \alpha 2$, and $\beta 1$ genes, YAP and TAZ decreased in terms of mRNA and protein. Therefore, this experiment was the first to confirm that SLA surface microtopography facilitates osteoblast differentiation by regulating YAP/TAZ and confirms that the process can be related to integrins $\alpha 1, \alpha 2$, and $\beta 1$.

Keywords: SLA Ti implant; YAP; TAZ; MC3T3-E1 cell; osseointegration.

\section{Introduction}

Titanium (Ti) and its alloys are widely used in dental implants. Osseointegration, referring to the direct interface between the bone and implant, either structurally or functionally, is regarded as a prerequisite for a successful dental implant (1). Conducting surface modifications for implants can improve implant osseointegration (2). Therefore, bone formation or angiogenesis can be promoted by changing the implants' surface microtopography or surface chemical properties or by designing a drug delivery system to mediate adhesion, migration, 
proliferation, and differentiation. This process can also provide antibacterial properties that may improve longterm success rates (3-7). Airborne-particle abrasion, large-grit, acid-etched (SLA) technology is one of the most commonly used surface modification methods in commercially-available implants.

SLA technology refers to applying large-grit sand to blast the implant surface. This leads to the formation of first-step pits. Then, specific acids are used to etch the surfaces of the first-step pits to form second-step pits in an overlapped manner to blunt the sharp sandblasting edges and generate multi-step microtopography on the implant surface, thereby increasing the implants' surface roughness. The surface topography formed by SLA technology increases the implant surface at the micron level, facilitating protein adsorption and cell adhesion and inducing osteoblast differentiation to promote bone formation and osseointegration (8). Clinical retrospective surveys showed that SLA implants effectively increased the bone mass in the immediate and early loading periods and that the implants' survival rate was above $97 \%$ (2). Ten years after implantation, the implants' survival rate was as high as $99.7 \%$, whereas the incidence rate of peripheral inflammation was only $7 \%$ (9). Therefore, SLA technology provides beneficial clinical effects for promoting implant osseointegration and improving the implant success rate.

Yes-associated protein (YAP) and transcriptional coactivator with the PDZ-binding motif (TAZ) genes are ubiquitously expressed in multiple organs. The two proteins are transcriptional co-activators. They act in parallel to regulate cell proliferation and survival, organogenesis, and cancer development (10). YAP and TAZ in the cytoplasm are translocated into the cell nucleus under appropriate circumstances to exert effects on gene transcription. Research first identified YAP and TAZ as critical substrates of the Hippo signaling pathway, which negatively regulates cell proliferation and differentiation $(10,11)$.

Recently, studies addressed YAP's and TAZ's roles in affecting mesenchymal stem cells (MSCs) and osteoblast differentiation by regulating runt-related transcription factor 2 (Runx2) transcriptional activity. Cytoplasmic YAP and TAZ translocate into the cell nucleus in response to a specific extracellular matrix (ECM), further exerting transcriptional coactivation of Runx2 and repressing peroxisome proliferator activated receptor gamma (PPAR $\gamma)$, representing an osteogenesis differentiation-related gene $(12,13)$. Improvement of bone mineral density and enhancement of bone mass were also observed. Both YAP and TAZ are involved in regulating cell differentiation in primary osteoblasts and MC3T3-E1 cells $(14,15)$.

Surface topography mediates YAP's and TAZ's contributions to differentiation of MSCs, which has been proven in the tissue engineering field. In response to micromorphology, YAP and TAZ induce cells to differentiate into osteoblasts. Microtopography and nanotopography, fabricated by different materials and treatments, exert distinct regulations on YAP and TAZ (15-17). However, the mechanism of SLA technology for commercial surface modification of dental implants remains elusive.

In terms of SLA microtopography, Olivares-Navarrete, Lai, Agarwal, and Gittens et al. $(6,18-20)$ determined that integrins $\alpha 1, \alpha 2$, and $\beta 1$ can promote osteoblast differentiation. Tang found that integrin could regulate YAP/ TAZ through the Rho GTP enzyme $(21,22)$. Therefore, if SLA surface microtopography facilitates implant osseointegration by regulating YAP/TAZ, the process may be related to integrin.

\section{Materials and Methods \\ Ti preparation and surface characterization}

Ti-6Al-4V is sometimes called $\mathrm{TC} 4$, or $\mathrm{Cr} 5$ in the USA. As a classic implant material that is available on the market, its sound performance has been proven for a long time, with excellent biocompatibility and osseointegration properties, adequate yield strength and ultimate strength, and high dynamic strength $(23,24)$. In this experiment, rods of Ti6Al4V alloy were fabricated (Aecc Beijing Institute of Aeronautical Materials, Beijing, P. R. China) with diameters of $19.5 \mathrm{~mm}$ and were cut into $2.5-\mathrm{mm}$ thick disks. Each disk was polished to an average roughness of $0.2 \mathrm{~mm}$ and a thickness of $0.01 \mathrm{~mm}$. The SLA disks were manufactured with large grit $(250 \mu \mathrm{m})$ jetted onto machined Ti surfaces after immersion in a mixed solution of $18 \% \mathrm{HCL}$ and $48 \% \mathrm{H}_{2} \mathrm{SO}_{4}$ at $60^{\circ} \mathrm{C}$ for $30 \mathrm{~min}$. Ultrasonic cleaning occurred with concentrated cleaning solution (Micro90, International Products Corporation, New York, NY, USA), absolute ethyl alcohol, and deionized water, in sequence. Polished Ti and SLA Ti disks were steeped in $75 \%$ alcohol for $12 \mathrm{~h}$ and exposed to ultraviolet (UV) radiation for $1 \mathrm{~h}$. The surface topography of the Polished Ti and SLA Ti disks was examined with a field emission scanning electron microscope (FESEM) (SU-70, Hitachi, Tokyo, Japan). A Wyko NT9300 Optical Profiler (Veeco, New York, NY, USA) was used to examine the average surface roughness $(\mathrm{Ra})$ of the Polished Ti and SLA Ti disks. 


\section{MC3T3-E1 cell culture}

Osteoblast-like MC3T3-E1 cells (Cell Bank of the Chinese Academy of Sciences, Shanghai, P. R. China) were cultivated as previously described (25). The MC3T3-E1 cells were inoculated on the surface of the Polished Ti and SLA Ti disks, with a density of $4 \times 10^{4}$ cells, to assess the cellular localizations and expressions of YAP, TAZ, and integrins $\alpha 1, \alpha 2$, and $\beta 1$. The MC3T3-E1 cells were seeded on the surface of the SLA Ti disks at a density of $7 \times 10^{4}$ for the YAP, TAZ, and integrins $\alpha 1, \alpha 2$, and $\beta 1$ knockdown. The MC3T3-E1 cells for gene transfection were inoculated with $\alpha$-MEM containing fetal bovine serum (FBS). The MC3T3-E1 cells achieved $70-80 \%$ confluency and were pretreated with Opti-MEM (Invitrogen, Carlsbad, CA, USA). YAP, TAZ, and integrin $\alpha 1, \alpha 2$, and $\beta 1$-siRNA were transfected into cells with Lipofectamine 3000 (Invitrogen). Then, the cells were incubated in a $\mathrm{CO}_{2}$ incubator. After $6 \mathrm{~h}$, the medium was exchanged with $\alpha$-MEM containing FBS and the osteogenic supplement. The siRNA sequences are listed as follows:

Yap-1-siRNA: CGAGATGAGAGCACAGACA; Wwtr1-siRNA: CAGAATGACTTTAGAGAAT; integrin $\alpha 1$-siRNA: CCTTCTACATGTTGGATAA; integrin $\alpha 2$-siRNA: CCATCAATTTCCAAGCCTT; and integrin $\beta 1$-siRNA: GGAGAACCACAGAAGTTTA.

\section{Quantitative real-time reverse transcription polymerase chain reaction (qRT-PCR)}

After 3 days of incubation on the Polished Ti and SLA Ti, the mRNA expressions of YAP, TAZ, and integrins $\alpha 1, \alpha 2$, and $\beta 1$ were examined using qRT-PCR. After 48 $\mathrm{h}$ of YAP and TAZ transfection, the mRNA expression of Runx2 and bone sialoprotein (BSP) were analyzed with qRT-PCR. After $48 \mathrm{~h}$ of integrins $\alpha 1, \alpha 2$, and $\beta 1$ transfection, the mRNA expression of YAP and TAZ, and the transfection efficiency of YAP, TAZ, and integrins $\alpha 1, \alpha 2$, and $\beta 1$ were analyzed with qRT-PCR. QRT-PCR analysis was performed using a previously described protocol (25). The gene expression was normalized with glyceraldehyde-3-phosphate dehydrogenase (GAPDH) expression. The qRT-PCR primers were as follows:

YAP: 5'-TGAGATCCCTGATGATGTACCAC-3' and 5'-TGTTGTTGTCTGATCGTTGTGAT-3';

TAZ: 5'-GACAAGGCAAGCAAGACGTG-3' and 5'-GCTGAAAGCAACAGCAGTCC-3';

ITGA1: 5'-CGTGTCATCTCCTTCCCTCG-3' and 5'-AATAGCCAGCTCTCGTTTCCG-3';

ITGA2: 5'-TGGTAGTTGTGACCGATGGC-3' and 5'-ACCCAAGAACTGCTATGCCG-3';

ITGB1: 5'-CGGACGCTGCGAAAAGATGAA-3' and
5'-CCGCAAGATTTGGCATTTGCT-3';

Runx2: 5'-CCCAGCCACCTTTACCTACA-3' and 5'-TATGGAGTGCTGCTG GTCTG-3'; BSP: 5'-CAGGGAGGCAGTGACTCTTC-3' and 5'-AGTGTGGAAAGTGTGGCGTT-3'; and GAPDH: 5'-AGGTCGGTGTGAACGGATTTG-3' and 5'-TGTAGACCATGTAGTTGAGGTCA-3'.

\section{Immunofluorescence staining}

To assess YAP's and TAZ's translocation from the cytoplasm into the nucleus, the MC3T3-E1 cells were incubated on the Polished Ti and SLA Ti disks for $24 \mathrm{~h}$. The cells were fixed with $4 \%$ paraformaldehyde for 15 min and permeabilized with $0.5 \%$ Triton X-100 for 20 $\min$. The nonspecific antibody interaction was blocked with goat serum for $30 \mathrm{~min}$. The disks were incubated with rabbit monoclonal antibody to YAP (1:100 dilution; Cell Signaling Technology, Boston, MA, USA) and rabbit polyclonal antibody to TAZ (1:100 dilution; Abcam, Cambridge, UK) overnight at $4^{\circ} \mathrm{C}$. Then they were incubated with a FITC-labeled goat anti-rabbit IgG (1:50 dilution; Jackson Immuno Research, Baltimore, MD, USA) to visualize the primary antibodies at $37^{\circ} \mathrm{C}$ for $50 \mathrm{~min}$. The nuclei were counterstained with $4^{\prime}, 6$ diamidino-2-phenylindole (DAPI, Invitrogen) for $5 \mathrm{~min}$. The disks were rinsed with PBS buffer in the interval of each of the two reagents. YAP's and TAZ's translocation was visualized and photographed (LSM 780 Spectral Confocal Laser Scanning microscope, Zeiss, Jena, Germany).

\section{Protein extraction and Western blot analysis}

The protein expression of YAP, TAZ, and integrins $\alpha 1$, $\alpha 2$, and $\beta 1$ on the Polished Ti and SLA Ti after 3 days, the protein expression of Runx 2 and BSP after $48 \mathrm{~h}$ of YAP and TAZ transfection, and YAP's and TAZ's protein expression after $48 \mathrm{~h}$ of integrins $\alpha 1, \alpha 2$, and $\beta 1$ transfection were examined with Western blot analyses using a previously described protocol (25). The membrane was incubated overnight at $4{ }^{\circ} \mathrm{C}$ with rabbit monoclonal antibody to YAP; rabbit polyclonal antibody to TAZ; rabbit monoclonal antibody to Runx2 (1:1,000 dilution; Cell Signaling Technology, Boston, MA, USA); rabbit polyclonal antibody to BSP (1:500 dilution; Cell Signaling Technology); rabbit polyclonal antibody to ITGA1 (1:1,000 dilution; Affinity, Cincinnati, OH, USA); rabbit monoclonal antibody to ITGA2 (1:1,000 dilution; Abcam, Cambridge, Britain); rabbit polyclonal antibody to ITGB1 (1:500 dilution; Wanleibio, Shenyang, Liaoning, P. R. China); or rabbit polyclonal antibody to GAPDH (1:10,000 dilution; ProteinTech, Chicago, IL, USA). The 


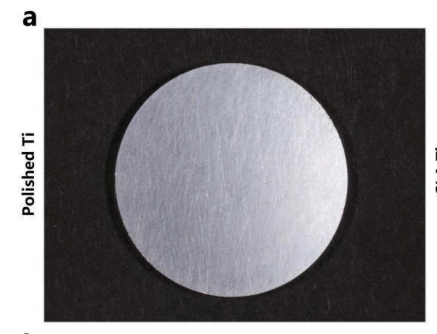

b
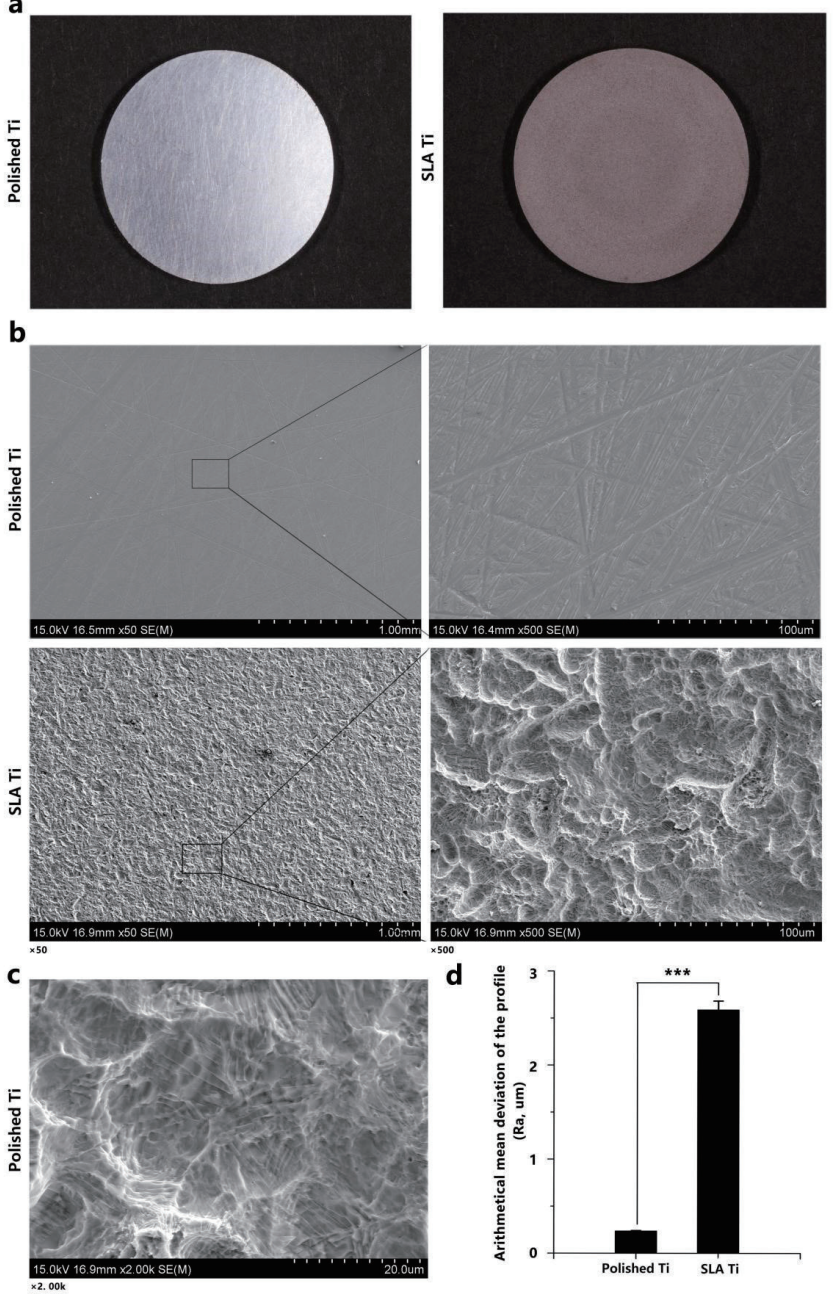

d

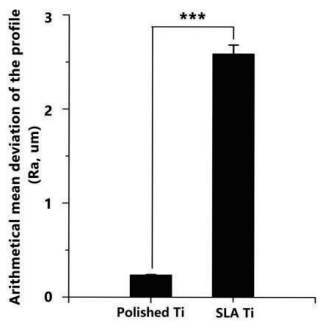

Fig. 1 Images of Polished $\mathrm{Ti}$ and sandblasted, large-grit, acidetched (SLA) Ti under different power lenses: (a) General image of Polished Ti and SLA Ti; (b) surface topography images of Polished Ti and SLA Ti under scanning electron microscope $(\mathrm{SEM})$ with low-power $(\times 50)$ and high-power $(\times 500)$ lenses; $(\mathrm{c})$ SLA Ti surface topography under SEM with high-power lens $(\times 2.00 \mathrm{k})$; (d) average surface roughness $(\mathrm{Ra})$ of Polished Ti and SLA Ti under an optic profiler; ${ }^{* * *} P<0.0001$ when SLA Ti and Polished Ti are compared, and the difference is statistically significant.

polyvinylidene difluoride membrane was rinsed with $1 \%$ TBST buffer and incubated with goat anti-rabbit IgG peroxidase conjugate at room temperature (RT) for $1 \mathrm{~h}$. The membrane, incubated with HRP-conjugated rabbit polyclonal antibody to $\beta$-actin (1:5,000 dilution; ProteinTech), was directly placed at RT for $1 \mathrm{~h}$. Immunodetection was performed by SageCapture software (Beijing SaiZhi Venture Technology Co., Ltd., Beijing, P. R. China) with an enhanced chemiluminescence kit.

\section{Statistical analysis}

Statistical software (SPSS 24.0, IBM Corp., Armonk, NY, USA) was used to analyze the experimental results, and Shapiro-Wilk test was used to analyze data normality. The test level was $\alpha=0.05$, and the data were in normal distribution $(P>0.05)$. The representative data of YAP, TAZ, Runx2, and BSP were presented as mean \pm standard deviation using statistical software (GraphPad Prism 5, GraphPad Software Inc., La Jolla, CA, USA). The $t$-test was used to detect differences between two groups, and one-way ANOVA (Dunnett's multiple comparisons test) was used to compare the mean of each group with a control group. Statistical significance was defined as $* P$ $<0.05, * * P<0.01$, and $* * * P<0.0001$.

\section{Results}

\section{Topography features of SLA}

After grinding and polishing, the smooth Ti6Al4V specimen appeared as a silver-white lustrous metal with scratches visible to the naked eye. The SLATi6Al4V specimen produced with SLA technology based on the previously produced smooth Ti specimen appeared as a gray, rough, and lackluster metal (Fig. 1a). After cleaning, the Polished Ti and SLA Ti underwent vacuum freezedrying followed by metal spraying. When observing the surface microtopography of the Ti plate with FESEM, the surface of the Polished Ti $(\times 50, \times 500)$ was relatively smooth with several scratches visible in different directions. After sandblasting and acid-etched treatment, the SLA Ti specimen $(\times 50, \times 500)$ acquired a large number of surface pits, which were continuously overlapped (Fig. 1b). Under a high magnification lens, the surface topography of the SLA Ti $(\times 2.0 \mathrm{k})$ showed first-step pits with a pit diameter of approximately $30 \mu \mathrm{m}$. There were several second-step pits with a pit diameter of approximately 4-8 $\mu \mathrm{m}$, and discontinuous, short scratches were still visible (Fig. 1c). To further observe the roughness of the two specimens, an optical profilometer was used to measure $\mathrm{Ra}$. The results were $0.2358 \pm 0.005199 \mu \mathrm{m}$ for $\mathrm{Ra}$ of the Polished Ti and $2.587 \pm 0.09597 \mu \mathrm{m}$ for Ra of the SLA Ti; thus, the Ra of the SLA Ti was significantly higher than that of the Polished Ti $(P<0.0001)$ (Fig. 1d).

\section{SLA induced the translocation of YAP and TAZ}

To confirm that the surface topography of SLA technology was conducive to YAP/TAZ's nuclear migration, the MC3T3-E1 cells were cultured on the Polished Ti and SLA Ti for $24 \mathrm{~h}$. Then, the distributions of the YAP/ TAZ proteins in the cells were observed, by immunofluorescence staining, in the nucleus and cytoplasm of Polished Ti, whereas the distribution in the cytoplasm was more widespread. On SLA Ti, the YAP proteins mainly clustered in the nucleus, and few were observed in the cytoplasm surrounding the nucleus. Nearly all the 

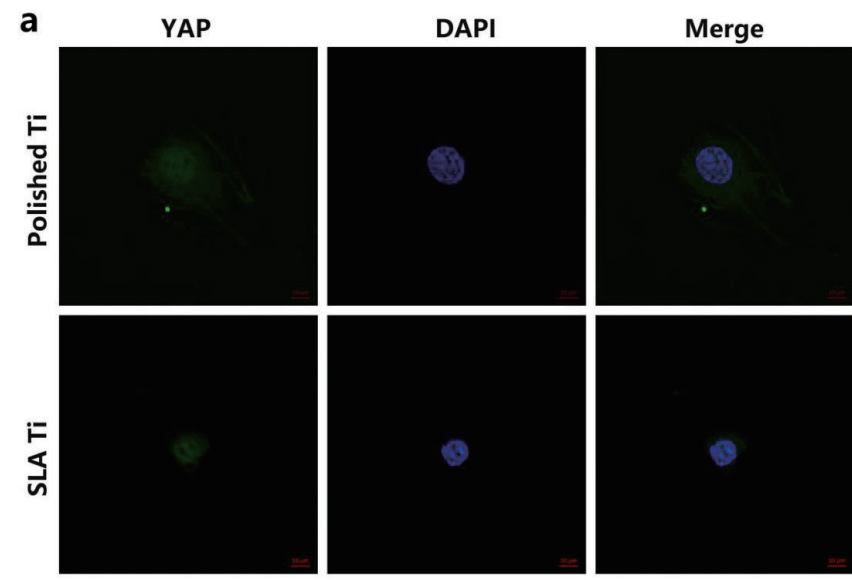

b

TAZ
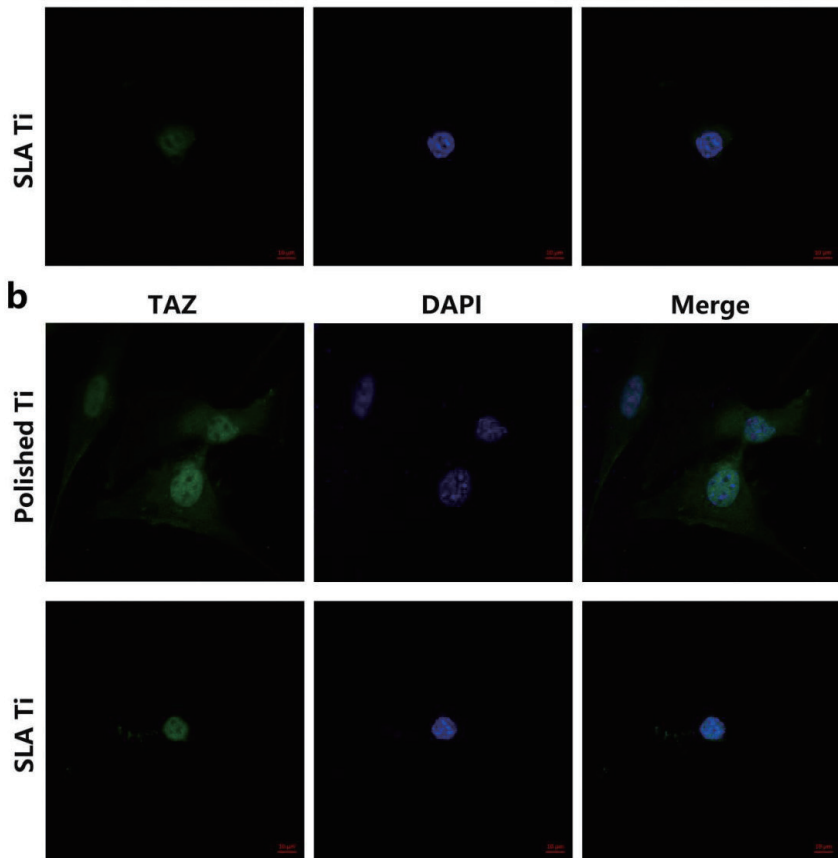

Merge
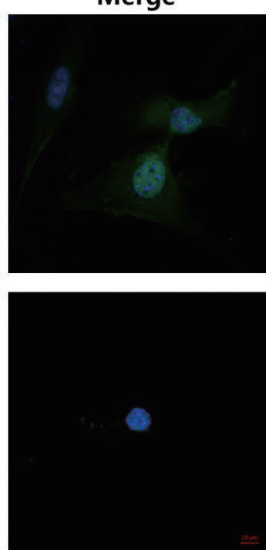

Fig. 2 Intracellular localization of Yes-associated protein (YAP) (a) and transcriptional coactivator with PDZ-binding motif (TAZ) (b) on Polished Ti and SLA Ti under immunofluorescent staining detection for $24 \mathrm{~h}$. "Merge" represents the blended image of the target protein (YAP/TAZ) and nucleus.

TAZ was gathered in the nucleus (Fig. 2). These results were influenced by the surface microtopography of SLA technology, and YAP/TAZ was transferred from the cytoplasm to the nucleus.

\section{SLA enhanced YAP and TAZ expression}

To further study SLA topography's influence on YAP/ TAZ expression in osteoblasts, qRT-PCR was used to detect changes in the mRNA levels of YAP/TAZ on Polished Ti and SLA Ti. For the MC3T3-E1 cells cultured for 3 days on the two materials, the results showed that the mRNA level of YAP on SLA Ti $(1.895 \pm 0.1548, P<$ $0.01)$ increased remarkably compared to that on Polished Ti $(1.000 \pm 0.08451)$. TAZ's mRNA level $(2.645 \pm$ $0.05891, P<0.0001)$ also increased compared to that on a Polished Ti (1.000 \pm 0.07173$)$, with both having statistical significance (Fig. 3a). Meanwhile, Western blot demonstrated that the SLA's surface microtopography also increased YAP/TAZ's protein level when compared a
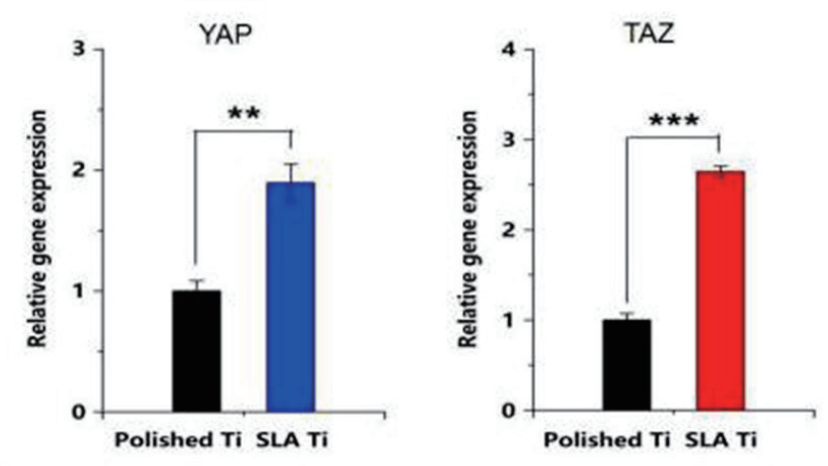

b
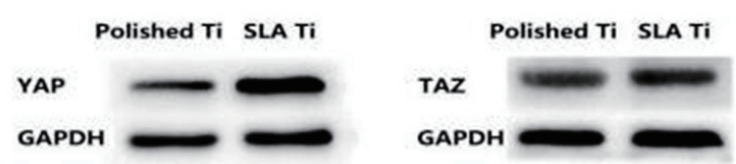

Fig. 3 mRNA expression and protein expression of YAP and TAZ on Polished Ti and SLA Ti: (a) mRNA expression after GAPDH standardization of YAP and TAZ on Polished Ti and SLA Ti, detected with qRT-PCR on the third day; (b) protein expression of YAP and TAZ on Polished Ti and SLA Ti, detected with Western blot on the third day; ${ }^{* * P}<0.01$ when SLA Ti and Polished Ti are compared, and the difference is statistically significant; $* * * P$ $<0.0001$ when SLA Ti and Polished Ti are compared, and the difference is statistically significant.

to Polished Ti (Fig. 3b). The above results indicated that SLA microtopography could increase YAP/TAZ expression in terms of the transcription.

\section{YAP and TAZ knockdown attenuated SLA-induced MC3T3-E1 osteogenic differentiation}

To explore the regulation of SLA microtopography over osteogenic differentiation by YAP and TAZ, the MC3T3-E1 cells were cultured on SLA Ti, and the genes yap 1 and wwtr 1 of YAP and TAZ were silenced through siRNA to observe changes in the osteogenic differentiation markers Runx2 and BSP. On the second day of YAP gene silencing, the mRNA level of Runx2 $(0.5808 \pm 0.04188, P<0.01)$ was significantly lower than that of the transfected nonspecific sequence group $(1.000 \pm 0.06271)$, while the mRNA level of BSP $(0.6345$ $\pm 0.05408, P<0.05)$ was also lower than that of the control group (1.000 \pm 0.06521$)$ (Fig. 4a). Similarly, after TAZ gene silencing, the mRNA level of Runx2 $(0.5640 \pm 0.01611, P<0.0001)$ was significantly lower than that of the control group $(0.9997 \pm 0.01729)$, and the mRNA level of BSP $(0.2363 \pm 0.02777, P<0.01)$ was also significantly lower than that of the control group $(1.000 \pm 0.06521)$ (Fig. 4b). The protein levels of Runx2 and BSP were further detected using Western blot. It was 
a

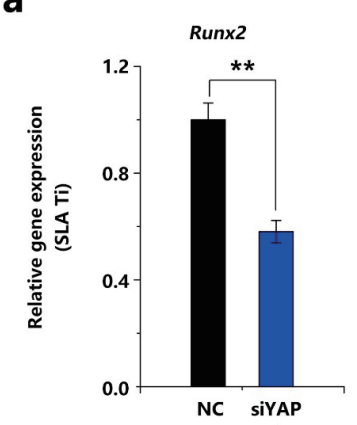

b

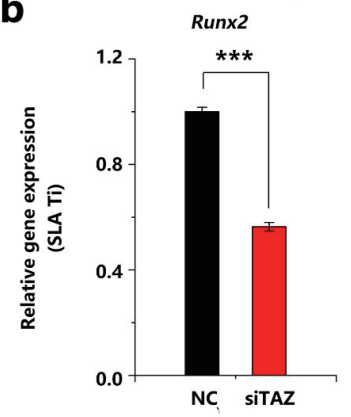

C

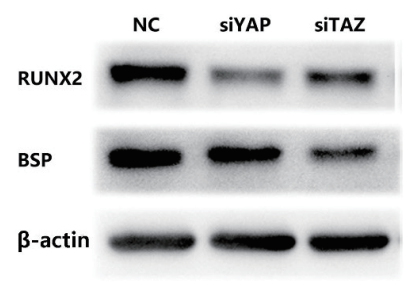

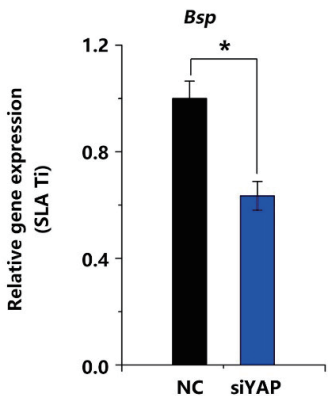

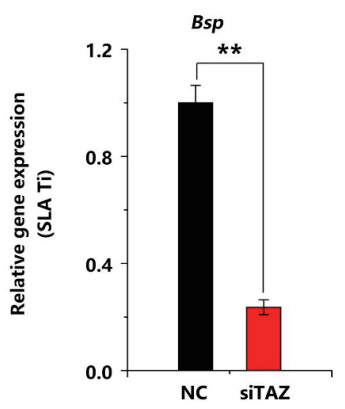

d

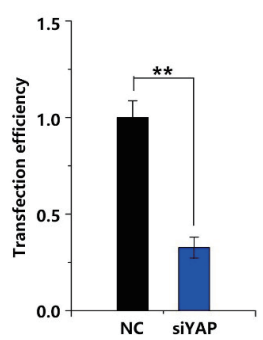

Fig. 4 Comparison of runt-related transcription factor 2 (Runx2) and bone sialoprotein (BSP) in different groups by qRT-PCR and Western blot: (a) mRNA expression after GAPDH standardization of Runx2 and BSP, detected with qRT-PCR on the 2nd day when YAP genes are silenced; (b) mRNA expression after GAPDH standardization of Runx2 and BSP, detected with qRT-PCR on the 2nd day, when TAZ genes are silenced; (c) mRNA expression after GAPDH standardization of Runx2 and BSP, detected with Western blot on the 2nd day when YAP and TAZ genes are silenced; (d) gene silencing efficiency of YAP and TAZ, detected with qRT-PCR on the 2 nd day. ${ }^{*} P<0.05$ is considered statistically significant; $* * P<0.01$ is considered statistically significant; $* * * P<0.0001$ is considered statistically significant.

discovered that, upon YAP silencing, Runx2's and BSP's protein levels were decreased; the same thing occurred upon TAZ silencing (Fig. 4c). Figure 4d shows that YAP's silencing efficiency on the second day of gene silencing was approximately $70 \%(P<0.01)$, while TAZ's was approximately $75 \%(P<0.05)$. The above results showed that when YAP and TAZ were silenced, both of the osteogenic differentiation markers Runx 2 and BSP in the cells were downregulated, which had statistical significance.

\section{SLA enhanced the expression of integrin $\alpha 1$, integrin $\alpha 2$, and integrin $\beta 1$}

QRT-PCR was used to detect changes in the mRNA levels of integrins $\alpha 1, \alpha 2$, and $\beta 1$ on Polished Ti and SLA Ti. After the MC3T3-E1 cells were cultured for 3 days on the two materials, results showed that the mRNA levels of integrins $\alpha 1, \alpha 2$, and $\beta 1$ on SLATi $(0.008952 \pm 0.0004110$; $\left.7.327 \mathrm{e}^{-006} \pm 2.241 \mathrm{e}^{-006} ; 0.02978 \pm 0.003258 ; P<0.05\right)$ increased remarkably compared to those on Polished Ti $\left(0.002926 \pm 0.0001434 ; 3.547 \mathrm{e}^{-005} \pm 8.474 \mathrm{e}^{-006} ; 0.01247\right.$ $\pm 0.003680, P<0.05$ ) (Fig. 5a). Meanwhile, Western blot demonstrated that SLA's surface microtopography also increased the protein levels of integrins $\alpha 1, \alpha 2$, and $\beta 1$ compared with (Fig. 5 b) above results indicated that SLA microtopography could increase the expression of integrins $\alpha 1, \alpha 2$, and $\beta 1$ in terms of the transcription. 
a
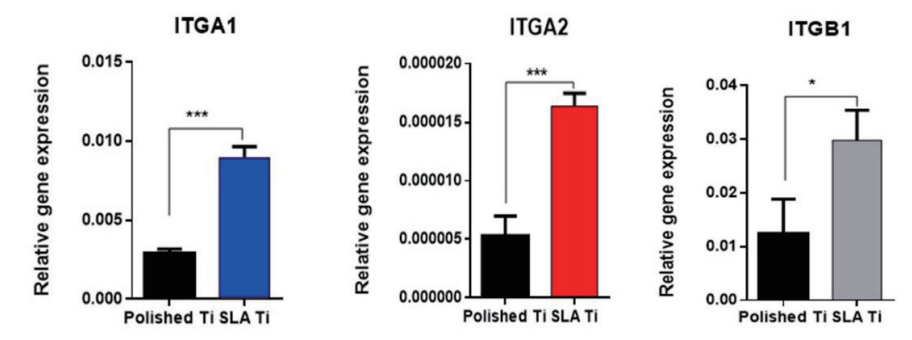

$\mathrm{b}$
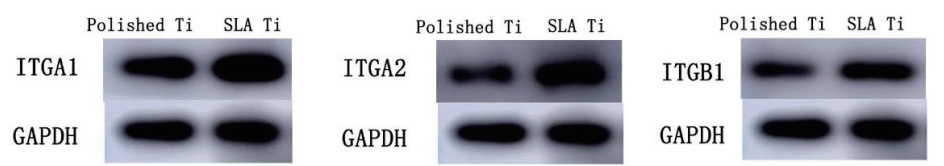

Fig. 5 mRNA and protein expression of integrins $\alpha 1, \alpha 2$, and $\beta 1$ on Polished Ti and SLA Ti: (a) mRNA expression after GAPDH standardization of integrin $\alpha 1$, integrin $\alpha 2$, and integrin $\beta 1$ on Polished Ti and SLA Ti, detected with qRT-PCR on the 3rd day; (b) protein expression of integrin $\alpha 1$, integrin $\alpha 2$, and integrin $\beta 1$ on Polished Ti and SLA Ti, detected with Western blot on the 3rd day; $* P<0.05$ when SLA Ti and Polished Ti are compared; $* * P<0.01$ is considered statistically significant when SLA Ti and Polished Ti are compared; ${ }^{* * *} P<$ 0.0001 is considered statistically significant when SLA Ti and Polished Ti are compared.
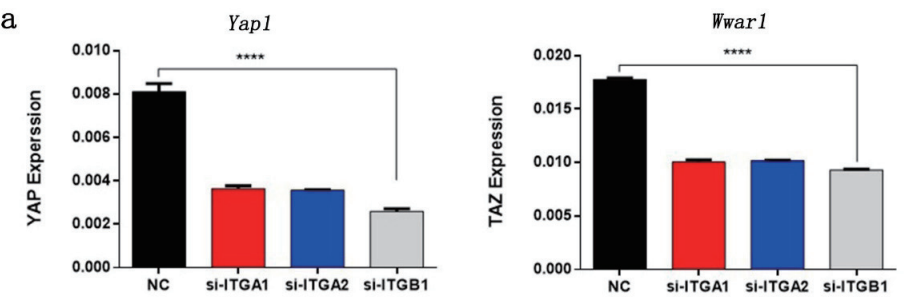

b

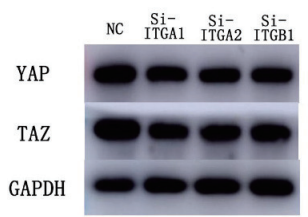

c

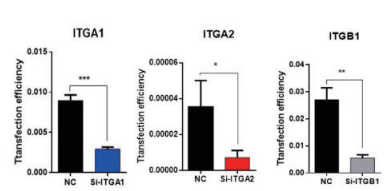

Fig. 6 Comparison of YAP and TAZ in different groups by qRT-PCR and Western blot: (a) mRNA expression after GAPDH standardization of YAP and TAZ, detected with qRT-PCR on the 2 nd day when integrin $\alpha 1$, integrin $\alpha 2$, and integrin $\beta 1$ genes were silenced; (b) protein expression after GAPDH standardization of YAP and TAZ, detected with Western blot on the 2nd day when integrin $\alpha 1$, integrin $\alpha 2$, and integrin $\beta 1$ genes were silenced; (c) gene silencing efficiency of integrin $\alpha 1$, integrin $\alpha 2$, and integrin $\beta 1$, detected with qRT-PCR on the 2 nd day. $* P<0.05$ is considered statistically significant; $* * P<0.01$ is considered statistically significant; $* * * P<0.0001$ is considered statistically significant.

\section{Integrins $\alpha 1, \alpha 2$, or $\beta 1$ knockdown attenuated}

\section{SLA-induced expression of YAP and TAZ}

To verify that SLA microtopography enhanced YAP and TAZ expression by integrins $\alpha 1, \alpha 2$, or $\beta 1$, MC3T3-E1 cells were cultured on SLA Ti, and the integrins $\alpha 1, \alpha 2$, or $\beta 1$ genes were silenced through siRNA to observe changes in YAP and TAZ expression. On the third day of gene silencing of integrins $\alpha 1, \alpha 2$, or $\beta 1$, mRNA levels of YAP and TAZ were significantly lower than those of the transfected nonspecific sequence groups $(P<0.05)$ (Fig. 6a). This study further detected the protein levels of YAP and TAZ using Western blot and discovered that upon integrins $\alpha 1, \alpha 2$, or $\beta 1$ being silenced, YAP's and TAZ's protein levels decreased (Fig. 6b). Figure 6c shows the silencing efficiency of integrins $\alpha 1, \alpha 2$, and $\beta 1$ on the second day of gene silencing. The above results showed that when integrins $\alpha 1, \alpha 2$, and $\beta 1$ were silenced, both YAP and TAZ in the cells were downregulated, which had statistical significance. 


\section{Discussion}

SLA technology is a commonly used surface modification method for dental implants. The hierarchical microtopography formed by SLA technology not only allows implant-osseous tissue to form proper mechanical chimerization, but also increases the superficial area of the implant-osseous tissue interface and further uplifts the material's surface roughness. This experiment investigated differences in SLA Ti and Polished Ti in surface microtopography with a scanning electron microscope and discovered that the SLA plate surface showed a hierarchical micropore morphology, with the pit diameter of the first-step pits being approximately $30 \mu \mathrm{m}$ and that of the second-step pits being approximately 3-8 $\mu \mathrm{m}$. Surface roughness is usually expressed with $\mathrm{Ra}$. In this experiment, an optical profilometer detected that SLA Ti's RA was approximately $2.6 \mu \mathrm{m}$ and that of Polished Ti was approximately $0.24 \mu \mathrm{m}$. This is consistent with the Ra of the optimum Ti alloy surface, 2-3 $\mu \mathrm{m}$, obtained by Olivares-Navarrete et al. (19).

Osteoblasts are sensitive to implants' surface microtopography. The microtopography of SLA implants can promote osteoblasts differentiation, thus facilitating bone formation around implants (26). Multiple signaling pathways are involved in regulating SLA microtopography over osteoblasts. $\alpha 2 \beta 1$ integrins may induce the release of TGF- $\beta 1$ by activating PKC, ultimately promoting osteoprotegerin expression (27). Yang et al. (28) found that the $\beta$-catenin/LRP5 pathway also participated in the process of SLA surface microtopography, promoting bone marrow-derived mesenchymal stem cell osteogenic differentiation. Rho-associated coiled-coil forming protein kinase (ROCK) downstream of RhoA also mediated the SLA microtopography process, promoting osteoblast differentiation by activating the $\mathrm{Wnt} / \beta$-catenin pathway (29).

Both YAP and TAZ are transcriptional co-activators in mammalian cells. The hardness of the extracellular matrix and surface microtopography can promote MSC and osteoblast differentiation through regulating YAP/ TAZ inner cellular localization (30). The relatively hard extracellular matrix can promote YAP/TAZ nuclear migration and facilitate osteoblast and MSC differentiation. Previous research showed that YAP/TAZ migrated into the nucleus under the effect of specific surface microtopographies and combined with Runx2, promoting MSCs' osteoblast differentiation.

Pan et al. (31) adopted sandblasting to prepare the pure Ti surface micron structure and used alkali heat treatment to prepare the pure $\mathrm{Ti}$ surface micron-nano structure. They observed that, compared to the surface of Polished Ti, the micron-structured pure Ti surface could promote YAP migration into the nucleus, and YAP migration on a micron-nano multilevel structure surface was more obvious. Furthermore, the YAP content was also increased. In this experiment, immunofluorescent staining results showed that SLA microtopography could promote YAP and TAZ migration from the cytoplasm into the nucleus. Meanwhile, qRT-PCR and Western blot experimental results showed that the mRNA and protein expression of YAP/TAZ in cells on SLA Ti significantly increased compared to cells on Polished Ti. Therefore, SLA microtopography could further change intranuclear signaling pathways by increasing YAP/TAZ's intracellular content of and promoting their nuclear migration, influencing the osteoblasts' biological changes.

Runx2, also called core-binding factor a1 (Cbfa1), serves as the key transcription factor in osteoblast differentiation. Moreover, Runx2 is also the switch for osteoblast differentiation (32-35). BSP belongs to the family of small integrin-binding ligand N-linked glycoproteins and is one of the marker genes for osteoblast differentiation. BSP expression visibly increases in maturely differentiated osteoblasts, and BSP is highly expressed in the early stage of bone deposition (36). BSP can also regulate the formation of osteoclasts. The lack of BSP influences the function of osteoblasts and osteoclasts (37). BSP knockdown mouse models have shown a relatively low level of bone remodeling for lowered osteoblast and osteoclast activity (38).

This experiment adopted siRNA to inhibit YAP/TAZ in MC3T3-E1 cells on SLA microtopography surfaces. Then, qRT-PCR and Western blot were used to detect the expression of the osteoblast marker genes Runx2 and BSP. The results showed that the mRNA and protein levels of Runx2 and BSP obviously declined after YAP or TAZ silencing. This indicated that YAP/TAZ could promote the expression of Runx2 and BSP on the SLA microtopography surface, which was consistent with the above conclusion of regulating YAP/TAZ to promote MSC osteoblast differentiation in the microtopography prepared by histological engineering (16).

In terms of SLA microtopography, $\alpha 1 \beta 1$ and $\alpha 2 \beta 1$ integrin may promote osteoblast differentiation $(3,18$ 20). Integrin can form physical connections with ECM and cytoplasm fibers on cell membranes through mutual effects with various signaling proteins, namely, focal adhesion. As the mechanical stimulation receptor on the bone tissue cell surface, focal adhesion-cytoskeleton plays an important role in cell adhesion, survival, proliferation, migration, and invasion $(39,40)$. Pan et al. (31) found that the multilevel structure microtopography 
surface generated effects on myosin via the integrin pathway, thus changing the cytoskeleton's tension. The mechanical conduction caused by the tension promoted YAP/TAZ's nuclear migration and combined them with the transcription factors Runx2 and PPAR $\gamma$ inside the nucleus, determining the BMSCs osteoblast or fat differentiation. This study found that SLA microtopography enhanced the expression of integrin $\alpha 1$, integrin $\alpha 2$, and integrin $\beta 1$. After the integrin $\alpha 1, \alpha 2$, and $\beta 1$ genes were silenced, YAP and TAZ decreased in terms of mRNA and protein, suggesting that integrins $\alpha 1, \alpha 2$, and $\beta 1$ participate in the process of YAP/TAZ regulating the differentiation of osteoblasts on SLA microtopography surface.

The Rho GTP enzyme, downstream of integrin, takes part in the composition of focal adhesion. It belongs to the small $\mathrm{G}$ protein superfamily. It was recently proven that integrin can regulate YAP/TAZ through the Rho GTP enzyme. Tang et al. discovered that MT1-MMP (membrane-type 1 matrix metalloproteinase) could activate the Rho GTP enzyme through $\beta 1$ integrin, enabling YAP/TAZ's nuclear migration and promoting skeletal stem cell osteoblast differentiation (22). On the surface of TNTs, focal adhesion kinase, downstream of integrin in the MC3T3-E1 cells, could regulate YAP through RhoA (17). The Rho GTP enzyme also regulated YAP by mechanical stress or cytoskeleton tension (21). Therefore, this study determined that the microtopography, formed by SLA technology on the Ti surface, changed cell spreading and form, activated integrins on the cell surface, conducted focal adhesion assembly, and activated the Rho GTP enzyme through a series of kinases. Meanwhile, the change of the cell form also formed micro-mechanical acting forces due to the change in cytoskeleton tension, which similarly worked on the Rho GTP enzyme. The activated Rho GTP enzyme may regulate Runx2 transcriptional activity through regulating YAP/TAZ's nuclear migration, thus promoting osteoblast differentiation.

\section{Acknowledgments}

Titanium specimens were fabricated with the assistance of Shandong Maier Medical Technology Co., Ltd. This work was supported by the Natural Science Foundation of Shandong Province (No. ZR2014HM053).

\section{Conflict of interest}

The authors declare that they have no competing interest.

\section{References}

1. Kim MH, Park K, Choi KH, Kim SH, Kim SE, Jeong CM et al. (2015) Cell adhesion and in vivo osseointegration of sandblasted/acid etched/anodized dental implants. Int J Mol Sci 16, 10324-10336.

2. Chambrone L, Shibli JA, Mercúrio CE, Cardoso B, Preshaw PM (2015) Efficacy of standard (sla) and modified sandblasted and acid-etched (slactive) dental implants in promoting immediate and/or early occlusal loading protocols: a systematic review of prospective studies. Clin Oral Implan Res 26, 359-370.

3. Ross AM, Jiang ZX, Bastmeyer M, Lahann J (2012) Physical aspects of cell culture substrates: topography, roughness, and elasticity. Small 8, 336-355.

4. Gu Z, Zhang X, Li L, Wang Q, Yu X, Feng T (2013) Acceleration of segmental bone regeneration in a rabbit model by strontium-doped calcium polyphosphate scaffold through stimulating VEGF and bFGF secretion from osteoblasts. Mater Sci Eng C Mater Biol Appl 33, 274-281.

5. Jin GD, Cao HL, Qiao YQ, Meng FH, Zhu HQ, Liu XY (2014) Osteogenic activity and antibacterial effect of zinc ion implanted titanium. Colloid Surface B 117, 158-165.

6. Agarwal R, García AJ (2015) Biomaterial strategies for engineering implants for enhanced osseointegration and bone repair. Adv Drug Deliver Rev 94, 53-62.

7. Zhou JH, Zhao LZ (2016) Multifunction Sr, Co and F co-doped microporous coating on titanium of antibacterial, angiogenic and osteogenic activities. Sci Rep 6, 29069.

8. Bang SM, Moon H, Kwon YD, Yoo JY, Pae A, Kwon IK (2014) Osteoblastic and osteoclastic differentiation on SLA and hydrophilic modified SLA titanium surfaces. Clin Oral Implan Res 25, 831-837.

9. Van Velzen FJ, Ofec R, Schulten EA, Ten Bruggenkate CM (2015) 10-year survival rate and the incidence of peri-implant disease of 374 titanium dental implants with a SLA surface: a prospective cohort study in 177 fully and partially edentulous patients. Clin Oral Implan Res 26, 1121-1128.

10. Pan DJ (2010) The hippo signaling pathway in development and cancer. Dev Cell 19, 491-505.

11. Piccolo S, Dupont S, Cordenonsi M (2014) The biology of YAP/TAZ: hippo signaling and beyond. Physiol Rev 94, 1287-1312.

12. Keely PJ (2013) Proteolytic remodeling of the ECM and the geometric control of stem cell fate. Dev Cell 25, 325-326.

13. Xiao Z, Quarles LD (2015) Physiological mechanisms and therapeutic potential of bone mechanosensing. Rev Endocr Metab Dis 16, 115-129.

14. Kaneko K, Ito M, Naoe Y, Lacy-Hulbert A, Ikeda K (2014) Integrin alpha $\mathrm{V}$ in the mechanical response of osteoblast lineage cells. Biochem Biophys Res Commun 447, 352-357.

15. Zhang YY, Gong H, Sun Y, Huang Y, Fan Y (2016) Enhanced osteogenic differentiation of MC3T3-E1 cells on gridtopographic surface and evidence for involvement of YAP mediator. J Biomed Mater Res A 104, 1143-1152. 
16. Yang WL, Han WQ, He W, Li J, Wang J, Feng H et al. (2016) Surface topography of hydroxyapatite promotes osteogenic differentiation of human bone marrow mesenchymal stern cells. Mater Sci Eng C Mater Biol Appl 60, 45-53.

17. Zhang H, Cooper LF, Zhang XN, Zhang Y, Deng F, Song JL et al. (2016) Titanium nanotubes induce osteogenic differentiation through the FAK/RhoA/YAP cascade. Rsc Adv 6, 44062-44069.

18. Gittens RA, Olivares-Navarrete R, Hyzy SL, Sandhage K H, Schwartz Z, Boyan B D (2014) Superposition of nanostructures on microrough titanium-aluminum-vanadium alloy surfaces results in an altered integrin expression profile in osteoblasts. Connect Tissue Res 55, 164-168.

19. Olivares-Navarrete R, Hyzy SL, Berg ME, Schneider JM, Hotchkiss K, Schwartz Z et al. (2014) Osteoblast lineage cells can discriminate microscale topographic features on titanium-aluminum-vanadium surfaces. Ann Biomed Eng 42, 2551-2561.

20. Olivares-Navarrete R, Rodil SE, Hyzy SL, Dunn G R, Almaguer-Flores A, Schwartz Z et al. (2015) Role of integrin subunits in mesenchymal stem cell differentiation and osteoblast maturation on graphitic carbon-coated microstructured surfaces. Biomaterials 51, 69-79.

21. Tang Y, Rowe RG, Botvinick EL, Kurup A, Putnam AJ, Seiki $M$ et al. (2013) MT1-MMP-dependent control of skeletal stem cell commitment via a beta 1 -integrin/YAP/TAZ signaling axis. Dev Cell 25, 402-416.

22. Driscoll TP, Cosgrove BD, Heo SJ, Shurden ZE, Mauck RL (2015) Cytoskeletal to nuclear strain transfer regulates YAP signaling in mesenchymal stem cells. Biophys J 108, 2783 2793.

23. Velasco-Ortega E, Jos A, Cameán AM, Pato-Mourelo J, Segura-Egea JJ (2010) In vitro evaluation of cytotoxicity and genotoxicity of a commercial titanium alloy for dental implantology. Mutat Res 702, 17-23.

24. Buser D, Saulacic N, Bosshardt DD, Bornstein MM, Berner S (2012) Bone apposition to a titanium-zirconium alloy implant, as compared to two other titanium-containing implants. Eur Cell Mater 23, 273-286.

25. Li JY, Liu SG, Xiao GN, Mao MY, Zhang XW, Sun HQ (2017) Fibroblast growth factor receptor 1 propagates estrogen and fluid shear stress driven proliferation and differentiation response in mc3t3-el cells. Mol Biol 51, 342-355.

26. Zinger O, Zhao G, Schwartz Z, Simpson J, Wieland M, Landolt D et al. (2005) Differential regulation of osteoblasts by substrate microstructural features. Biomaterials 26, 1837 1847.

27. Schwartz Z, Olivares-Navarrete R, Wieland M, Cochran DL, Boyan BD (2009) Mechanisms regulating increased production of osteoprotegerin by osteoblasts cultured on microstructured titanium surfaces. Biomaterials 30, 33903396.
28. Yang GL, Fang W, Liu T, He F, Chen X, Zhou Y et al. (2016) Gene expression profiling of bone marrow-derived stromal cells seeded onto a sandblasted, large-grit, acid-etched-treated titanium implant surfagce: the role of the Wnt pathway. Arch Oral Biol 61, 71-78.

29. Galli C, Piemontese M, Lumetti S, Macaluso GM, Passeri G (2012) Actin cytoskeleton controls activation of wnt/betacatenin signaling in mesenchymal cells on implant surfaces with different topographies. Acta Biomater 8, 2963-2968.

30. Kuroda M, Wada H, Kimura Y, Ueda K, Kioka N (2017) Vinculin promotes nuclear localization of TAZ to inhibit ECM stiffness-dependent differentiation into adipocytes. J Cell Sci 130, 989-1002.

31. Pan H, Xie Y, Zhang Z, Li K, Hu D, Zheng X et al. (2017) YAP-mediated mechanotransduction regulates osteogenic and adipogenic differentiation of BMSCs on hierarchical structure. Colloids Surf B Biointerfaces 152, 344-353.

32. Ortuno MJ, Susperregui ARG, Artigas N, Rosa JL, Ventura, F (2013) Osterix induces Collal gene expression through binding to $\mathrm{Sp} 1$ sites in the bone enhancer and proximal promoter regions. Bone 52, 548-556.

33. Takarada T, Nakazato R, Tsuchikane A, Fujikawa K, Iezaki T, Yoneda Y et al. (2016) Genetic analysis of Runx2 function during intramembranous ossification. Development 143, 211-218.

34. Perez-Campo FM, Santurtun A, Garcia-Ibarbia C, Pascual MA, Valero C, Garcés C et al. (2016) Osterix and RUNX2 are transcriptional regulators of sclerostin in human bone. Calcif Tissue Int 99, 302-309.

35. Zheng LL, Tu QS, Meng S, Zhang L, Yu L, Song J et al. (2017) Runx2/DICER/miRNA pathway in regulating osteogenesis. J Cell Physiol 232, 182-191.

36. Wade-Gueye NM, Boudiffa M, Vanden-Bossche A, Laroche N, Aubin JE, Vico L et al. (2012) Absence of bone sialoprotein (BSP) impairs primary bone formation and resorption: the marrow ablation model under PTH challenge. Bone 50, 1064-1073.

37. Wade-Gueye NM, Boudiffa M, Laroche N, Vanden-Bossche A, Fournier C, Aubin JE et al. (2010) Mice lacking bone sialoprotein (BSP) lose bone after ovariectomy and display skeletal site-specific response to intermittent PTH treatment. Endocrinology 151, 5103-5113.

38. Malaval L, Wade-Gueye NM, Boudiffa M, Fei J, Zirngibl R, Chen F et al. (2008) Bone sialoprotein plays a functional role in bone formation and osteoclastogenesis. J Exp Med 205, 1145-1153.

39. Wozniak MA, Modzelewska K, Kwong L, Keely PJ (2004) Focal adhesion regulation of cell behavior. Biochim Biophys Acta 1692, 103-119.

40. Schwartz MA (2010) Integrins and extracellular matrix in mechano-transduction. Csh Perspect Biol 2, a005066. 